\title{
Study of Wood Plastic Composites elastic behaviour using full field measurements
}

\author{
T. Ben Mbarek ${ }^{1,4}$, L. Robert ${ }^{2,3, a}$, F. Hugot ${ }^{5}$, J.-J.Orteu ${ }^{2,3}$, H. Sammouda ${ }^{1}$, A. Graciaa ${ }^{4}$, B. Charrier ${ }^{4}$ \\ ${ }^{1}$ LETTM, Ecole Supérieure des Sciences et de Technologie de H-Sousse, rue Lamine ElAbbassi, \\ 4011 H-Sousse, Tunisie \\ ${ }^{2}$ Université de Toulouse; INSA, UPS, Mines Albi, ISAE ; ICA (Institut Clément Ader); Campus \\ Jarlard, F-81013 Albi, France \\ ${ }^{3}$ Ecole des Mines Albi, Campus Jarlard, F-81013 Albi, France \\ ${ }^{4}$ Sylvadour, IUT des Pays de l'Adour, 371 rue du Ruisseau BP 20140004 Mont-de-Marsan, France \\ ${ }^{5}$ Université de Limoges, ENSIL, 16 rue Atlantis, Parc ESTER Technopole, BP 6804, 87068 \\ Limoges, France
}

\begin{abstract}
In this study, the mechanical properties and microstructure of HDPE/wood fibre composites are investigated. The four-point bending and tensile behaviour of Wood Plastic Composite (WPC) with or without additive are studied by using full-field strain measurements by 3-D Digital Image Correlation (3-D DIC). A non-linear behaviour is shown. The modulus of elasticity (MOE) is calculated as the tangent at zero strain of a Maxwell-Bingham model fitted onto experimental data. Four-point bending tests are analyzed thanks to the spatial standard deviation of the longitudinal strain field to determine the degree of heterogeneity. Cyclic tensile tests have been performed in order to analyze the damage of the material. Moreover, Scanning Electron Microscope (SEM) is used to characterize the morphology of the wood fibre/HDPE matrix interface for specimens with maleic anhydride modified polyethylene additive (MAPE).
\end{abstract}

\section{Introduction}

Since last decade, Wood Plastic Composites (WPC) have been a fast growing industry and decking represents the main part of their market in sectors such as garden furniture, automotive and buildings. WPC are widely used in USA. They are generally composed of a thermoplastic matrix and wood fibres [1, 2]. Both ecological aspects of wood materials and good low cost / mechanical properties ratio give great opportunities to these new products. However, more studies are required to evaluate and understand the WPC mechanical behaviour. Nzokou et al. [3] examined the relationship between stress wave non-destructive evaluation (NDE) modulus of elasticity (MOE) and static MOE determined by four-point bending test of commercial WPC and found that the stress wave NDE technique may not be appropriated to estimate the static MOE of wood plastic lumber. Adhikary et al. [4] studied the stability, the mechanical properties, and the microstructure of WPC from recycled or virgin high-density polyethylene (HDPE) with wood flour (Pinus radiata) as filler

\footnotetext{
a e-mail: Laurent.Robert@mines-albi.fr
} 
and showed that recycled HDPE and wood sawdust can be successfully used to produce stable and strong WPCs. Although that many studies are devoted to evaluating and understanding WPC mechanical behaviour from tensile and bending tests using crosshead displacement or mechanical extensometers, few studies used non-contact optical methods to analyze the mechanical performance of WPCs. Godora et al. [5] investigated the influence of additives on the global mechanical performances and the microscopic strain localization in PP-based WPC by Digital Image Correlation (DIC) coupled with tensile tests. Giving access to the full surface strain field of the specimen, the DIC technique allows a detailed investigation of complex micromechanical questions associated with the distribution of the strain in these heterogeneous materials.

In the present study, several HDPE based-WPC formulations have been characterized. Both tensile tests and 4-point bending tests have been carried out. As WPC are heterogeneous materials, the 3-D Digital Image Correlation (3-D DIC) technique [6], also known as stereo-correlation, is used for measuring the strain fields. Four-point bending longitudinal strain values are determined by calculating the strain mean values in an area located between the two upper loading rollers and in the lower and the upper parts of the vertical face of the bending sample. Stress values are determined considering the theory of elasticity. The MOE is then provided by a Maxwell-Bingham model fitted to the experimental stress / strain curves. The analysis of the local strain by 3-D DIC is also performed to study the strain heterogeneities evolution during these cycles. Furthermore, cyclic tensile tests have been performed in order to analyze the damage of the material. Finally, microstructure is analysed by SEM imaging.

In the sequel, the material and experimental methods are detailed. Then the four-point bending test results are presented and discussed. The next section is devoted to the results of the cyclic tensile tests. Last, the morphology of the fracture surface is analysed.

\section{Material and experimental method}

\subsection{Wood plastic composite material}

A commercial WPC is used for this study. Lacqtene HDPE (MFI=2,5 g/10min) polymer granulates are from Atofina and additives coupling used as compatibiliser is Maleic Anhydride Polyethylene (MAPE). Softwood pinus pinaster sawdust has morphological characteristics described in Table 1. Optical scanning device of fibre (MorFi LB01 system from Techpap) is used to determine the size distribution in frequency of the wood fibres. The very small elements (length $<30 \mu \mathrm{m}$ ), called fines, represent about $76 \%$ in total length and $17 \%$ of the total surface of all analyzed elements.

Table 1. Morphological characteristics and size distribution of wood fibres (pinus pinaster).

\begin{tabular}{c|c|c}
\hline Fibers & Size & Frequency \\
\hline Average length (without thin fibers) & $220 \mu \mathrm{m}$ & \\
Average thickness (without fines) & $30 \mu \mathrm{m}$ & \\
Curvature (without thin fibers) & $4.5 \%$ & $76 \%$ \\
Size distribution (in length \%) & Fines $<30 \mu \mathrm{m}$ & $9.7 \%$ \\
& $30-150 \mu \mathrm{m}$ & $6.5 \%$ \\
& $150-250 \mu \mathrm{m}$ & $1.5 \%$ \\
& $250-300 \mu \mathrm{m}$ & $2.5 \%$ \\
& $300-450 \mu \mathrm{m}$ & $1.3 \%$ \\
& $450-680 \mu \mathrm{m}$ & $1.2 \%$ \\
\hline
\end{tabular}

After wood fibre drying, premixed wood / HDPE pellets are done by extrusion process in a single screw device. Then $4 \times 10 \mathrm{~mm}^{2}$ section tensile and bending specimens are produced by injection. Parameters are set at: injection temperature $200^{\circ} \mathrm{C}$, zone $1160^{\circ} \mathrm{C}$, zone $2170^{\circ} \mathrm{C}$, zone 3 
$180^{\circ} \mathrm{C}$, mould temperature from $50^{\circ} \mathrm{C}$ to $70^{\circ} \mathrm{C}$; injection pressure 100 bars, injection speed $47 \mathrm{~mm} / \mathrm{s}$, speed of screw $60 \mathrm{~mm} / \mathrm{s}$; holding time $6 \mathrm{sec}$, cooling time $25 \mathrm{sec}$. Indexation of realised formulations of WPC with or without additive is presented in Table 2. Two formulations are studied, with or without MAPE agent: $10 \%$ wood content and $30 \%$ wood content.

Table 2. WPC formulations.

\begin{tabular}{l|l}
\hline Index & Description \\
\hline iWPCf10 & $10 \%$ wood without additive \\
iWPCf30 & $30 \%$ wood without additive \\
iaWPCf10 & $10 \%$ wood with $3 \%$ of MAPE additive \\
iaWPCf30 & $30 \%$ wood with $3 \%$ of MAPE additive \\
\hline
\end{tabular}

\subsection{Experimental methods}

\subsubsection{Tensile and 4-point bending}

Mechanical characterization was carried out onto a set of 5 specimens for each test using a universal testing machine device (INSTRON $5800 \mathrm{R}$ ) with a crosshead displacement velocity equal to 2 $\mathrm{mm} / \mathrm{min}$. Prior to testing, all specimens were conditioned at a temperature of $20{ }^{\circ} \mathrm{C}$. Tensile and 4point bending properties are tested according to standard guides dedicated for plastics and reinforced plastics composites, NF EN ISO 527-1-4 and NF EN ISO 178-1 respectively. Tensile specimen dimensions are $10 \mathrm{~mm}$ in width, $4 \mathrm{~mm}$ in thickness and $150 \mathrm{~mm}$ in length. Tensile strain measurements can be performed using a mechanical extensometer (50 $\mathrm{mm}$ gauge length), or by 3-D Digital Image Correlation (3-D DIC). Concerning the 4-point bending device used onto the universal testing machine, the two upper and lower rollers are respectively $26 \mathrm{~mm}$ and $60 \mathrm{~mm}$ spaced. Bending specimens have a length of $80 \mathrm{~mm}$ and a section of $4 \mathrm{~mm}$ (thickness) by $10 \mathrm{~mm}$.

It is known that due to the non-linear behaviour of WPC, mechanical properties such as MOE are difficult to obtain. In [7], the authors have concluded that the tangent MOE is the more appropriate to calculate tensile MOE. In this study, the non-linear strain/stress relationship is modelled, in the area where the stress remains monotonous, by the Maxwell-Bingham model:

$$
\sigma=a(1-\exp (-b \varepsilon))
$$

where $a$ and $b$ are parameters. A fitting is done between the experimental curve and the MaxwellBingham model. The MOE is then calculated as the first derivative at $\varepsilon=0$ of the fitted model.

\subsubsection{3-D Digital Image Correlation (3-D DIC)}

The 3-D DIC method is based on both digital image correlation (DIC) and stereovision [6]. For both tensile and bending tests, the 3-D DIC method is used to determine strain fields and strain mean values. For the tensile tests, the stereo-rig is composed of two 8 bit Qicam (Qimaging) CCD cameras allowing a 1360x1036 pixels resolution, equipped with Nikon $50 \mathrm{~mm}$ aperture 11 lenses. For the bending tests, two AVT Dolphin 145-B 1392x1040 pixels resolution cameras equipped with $100 \mathrm{~mm}$ zoom lenses are used. The calibration and numerical image processing are carried out with the assistance of the Vic-3 $\mathrm{D}^{\circledR}$ commercial software [8]. Because of the very small optical contrast of the fibres in the matrix, and in order to increase the resolution and spatial resolution, a random blackand-white speckle pattern was created at the surface of the specimen by using spray paint.

The 3-D DIC parameters of the tensile tests are as follow: region of interest (ROI) of $60 \times 10$ $\mathrm{mm}^{2}$, magnification factor of $16.5 \mathrm{px} / \mathrm{mm}$, subset size of $19 \times 19$ pixels $^{2}$ corresponding to a $1.18 \mathrm{~mm}$ displacement spatial resolution, strain spatial resolution (optical gauge size) of $1.5 \mathrm{~mm}(25 \times 25$ pixels $^{2}$ ) and acquisition frequency of $0.5 \mathrm{~Hz}$. The 3-D DIC parameters of the 4-point bending tests are: ROI of $4 \times 60 \mathrm{~mm}^{2}$, magnification factor of $30 \mathrm{px} / \mathrm{mm}$, subset size of $21 \times 21$ pixels ${ }^{2}$ 
corresponding to a $0.7 \mathrm{~mm}$ displacement spatial resolution, strain spatial resolution of $0.83 \mathrm{~mm}$ $\left(25 \times 25\right.$ pixels $\left.^{2}\right)$ and acquisition frequency of $0.2 \mathrm{~Hz}$. It has been shown in another study [9] that strain standard uncertainty can be estimated to about $10^{-4}$ in similar experimental conditions.

\section{Results and discussion}

\subsection{Four-point bending test results}

First, the "theoretical" and measured macroscopic strain values on the tension sample face are compared. The "theoretical" macroscopic strain is calculated knowing the measured load using the theory of elasticity, as [9]:

$$
\varepsilon=\frac{3 P(L-l)}{2 b h^{2} E}
$$

where $P$ is the measured load, $b$ and $h$ respectively the width and the height of the specimen, $L$ and $l$ respectively the distances between the lower and the upper loading rollers and $E$ the tensile MOE estimated in a previous work [10]. As the mechanical strain field is heterogeneous, macroscopic values of the longitudinal strains are thus determined by calculating the strain mean values of the 3D DIC measured strain field in a area located between the two upper loading rollers, and in the lower (max. positive strain) and the upper (min. negative strain) parts of the vertical face of the bending sample [9]. In practice, strain value on the tension sample face is the mean value of the measured strain along a line in the lower part of the vertical face, as it can be seen in Figure 1.

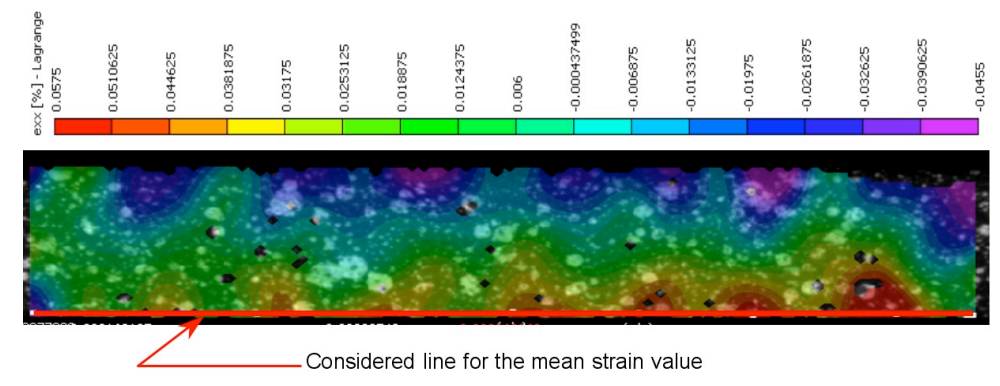

Fig. 1. Longitudinal strain field measured by 3-D DIC for iWPCf10 formulation. Definition of the line for calculating the mean strain value in traction for 4-point bending specimens.

Figure 2 presents the evolution of the strain function of time for the iWPCf10 formulation, for both cases: tensile strain evaluated from the theory of elasticity and load measurements, and tensile strain measured on the tension sample face by 3-D DIC, as previously discussed. As it can be clearly seen in Figure 2, strain values differ rapidly right from the start of the bending test. Thus the WPCs in 4-point bending show rapidly a non-linear macroscopic behaviour (divergence in relation to a linear elastic behaviour). By carefully observing the 3-D DIC strain field of Figure 1, it is worth pointing out that high strain domains appears on the vertical face close to both the tension sample face or the compression sample face.

One can reflect that WPC is prone to present damage which causes the non-linearity of the calculated strain. The strain map of Figure 1 shows that this damage corresponds most likely physically to a multi-crack, because the mode of damage appears for very small deformations, showing that the apparent strain heterogeneities are not so linked to the difference in modulus between the woof fibres and the HDPE matrix, but is mainly a consequence of local microscopic fractures between the fibres and the matrix [10]. 


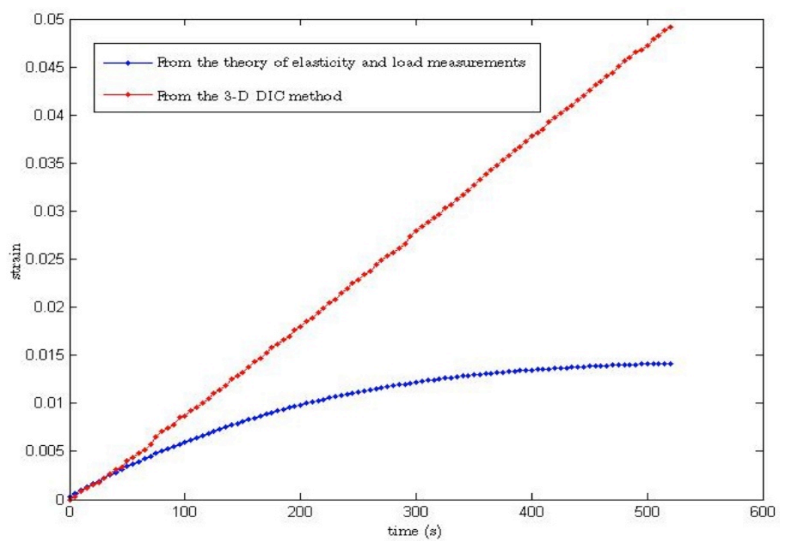

Fig. 2. Evolution of iWPCf10 four-point bending strain values on the tension sample face. Comparison between the values calculated (blue line) and measured by 3-D DIC (red line).

The theory of elasticity is applied to calculate the tensile nominal strength $\sigma$ on the tension sample face and the compressive nominal strength $-\sigma$ on the compression sample face, as [10]:

$$
\sigma=\frac{3 P(L-l)}{2 b h^{2}}
$$

The corresponding positive and negative strain values are respectively the mean value of the 3D DIC measured strain along a line in the lower (see Figure 1) and the upper parts of the vertical face of the bending sample. For each WPC formulation and experiments, strain / stress curves have been plotted and the MOE evaluated as discussed previously in Section 2.2.1. All the tensile and 4point bending MOE values are reported in Table 3 for the four formulations.

Table 3. Modulus of elasticity (MOE). Values in brackets are the standard deviation.

\begin{tabular}{|l|cc|c|}
\hline & \multicolumn{2}{|c|}{ 4-point bending tests } & Tensile tests \\
& Compression & Tensile & \\
\hline iWPCf10 & 1230 & 880 & 910 \\
MOE (MPa) & $(100)$ & $(20)$ & $(25)$ \\
\hline iaWPCf10 & 1480 & 1105 & 1137 \\
MOE (MPa) & $(90)$ & $(17)$ & $(26)$ \\
\hline iWPCf30 & 1926 & 1586 & 1532 \\
MOE (MPa) & $(25)$ & $(70)$ & $(100)$ \\
\hline iaWPCf30 & 2470 & 1795 & 1794 \\
MOE (MPa) & $(14)$ & $(27)$ & $(26)$ \\
\hline
\end{tabular}

The values of MOE have been obtained using the 3-D DIC method for tensile tests and bending tests and also using extensometer measurements for tensile tests. In this study, all the values obtained for the 4-point bending tests are almost in the uncertainty range of those obtained from tensile tests. The results found in tensile and compression during 4-point bending tests and tensile tests show that the MOE increases with the wood percentage in both cases with or without MAPE. Moreover, results of Table 3 highlight that the coupling agent confers to the composite the upper modulus. Furthermore, it is found that the tensile MOE from the 4-point bending tests are in very good agreement with the ones obtained from direct tensile tests for the four formulations. These results demonstrate the ability to determine MOE using the 3-D DIC method for WPC even when considering the case of heterogeneously loaded structures like bending beams. However, it is worth noting that for the 4-point bending tests, an increase of about $33 \%$ on average is observed between the MOE in compression relatively to the one in tension. Bending and compressive properties are 
less dependent on adhesion quality than tensile ones, as adhesion plays a main role in stress transfer from the matrix to the fibres [7].

Another advantage offered by the 3-D DIC method is to determine the degree of heterogeneity. In this work, the degree of heterogeneity is analysed thanks to the standard deviation of the spatial strain field along the line discussed previously and presented in Figure 1. For a homogenous material, the longitudinal strain is constant along lines parallel to the neutral axis. Figure 3 presents the evolution of the 3-D DIC longitudinal mean strain and strain standard deviation along the line in traction function of time. The value of the strain standard deviation for $10 \%$ wood fibre is relatively important (approximately 10\% of the average strain value, Figure 3(a)) whereas for 30\% wood fibre the relative value of the strain standard deviation becomes more important (until $20 \%$ of the average strain value, Figure 3(b)). It is shown thus that the degree of heterogeneity increases with the wood fibre percentage and that the strain heterogeneity increases proportionally to the increase of average strain. As expected, the heterogeneity linked to the local damage (microscopic crack and decohesion between fibres and matrix) increases both with wood content and strain level but more in the tensile part than in the compressive part of the 4-point bending sample (Figure 1).

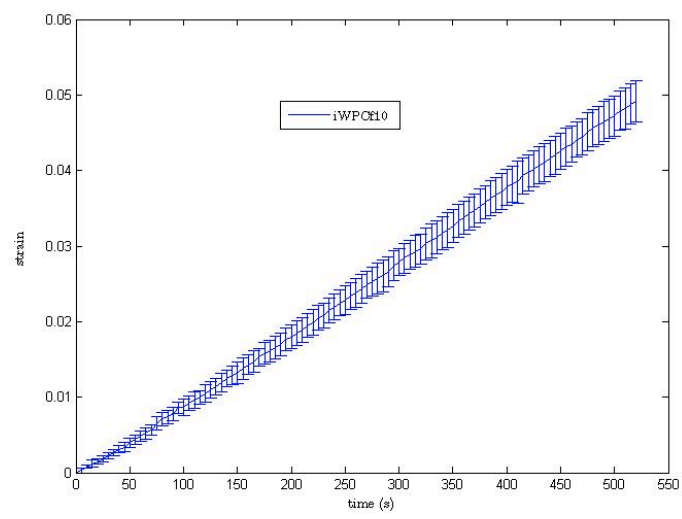

(a) iWPCf10 formulation

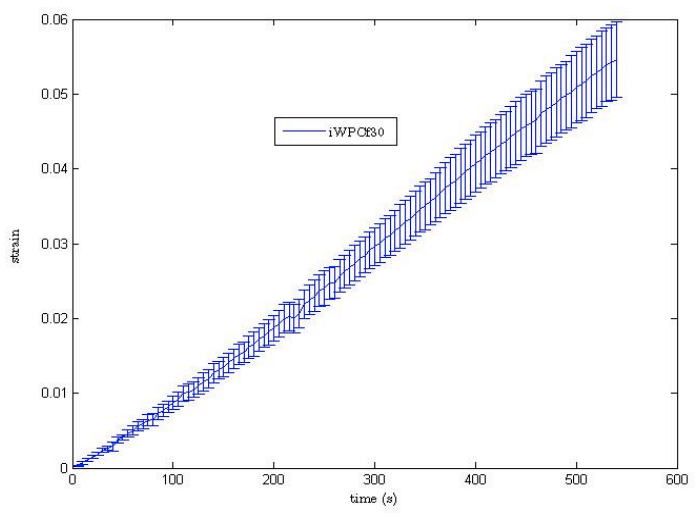

(b) iWPCf30 formulation

Fig. 3. Evolution of the 3-D DIC longitudinal mean strain versus time. Errors bars represent the value of the strain standard deviation.

\subsection{Cyclic tensile test results}

Cyclic tensile tests have been performed in order to analyse the composite damage. As a preliminary comment, it can be observed in Figure 4(a) that the strain both measured by the 3-D DIC method and with the mechanical extensometer are quite closer. However, an observation of Figure 4(a) in detail, shows that one can see a small difference between the two curves. This difference may come from the fact that (i) strains are not measured on the same face of the tensile test sample and (ii) the mechanical extensometer measurements are sensitive to the localisation of the extensometer rods. In fact, the 3-D DIC method gives a mean value of the strain field which takes into account all the local strain gradients which characterize the surface being considered. Thus, the 3-D DIC method allows to assess a macroscopic strain field less dependent on crack locations. The analysis of the local strain by 3 -D DIC is also performed to study the strain heterogeneities evolution during this cyclic tensile test.

The effect of the wood percentage injected with the HDPE matrix on the mechanical behaviour is treated in the case of iWPCf10 and iWPCf30 formulations. Tensile tests results performed with the both formulations are shown in Figure 4(b). The behaviour curves are characterised by a very restricted linear domain at the beginning of the tests. iWPCf10 linear domain is less important than iWPCf30. Elasticity of the WPC increases with wood fibre percentage. When the stress level 
increases, the behaviour moves to non-linear one. These results explain the global non linear behaviour of the WPC and for this reasons the MOE is evaluated according to the Maxwell-Bingham fit method.

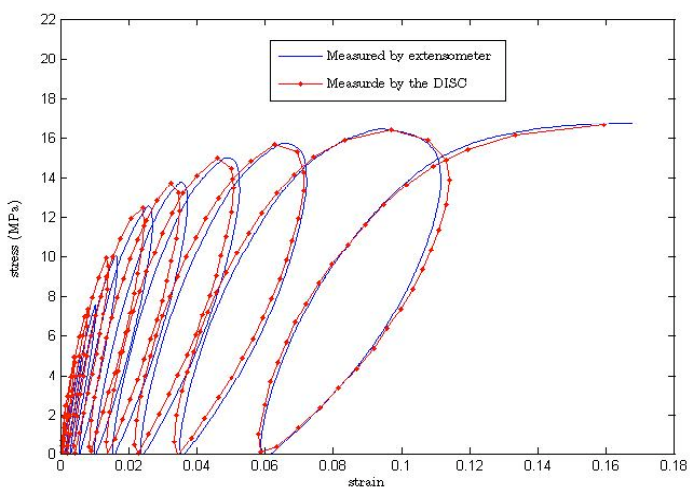

(a) iWPCf10 sample. Strain is measured by the mechanical extensometer and by 3-D DIC

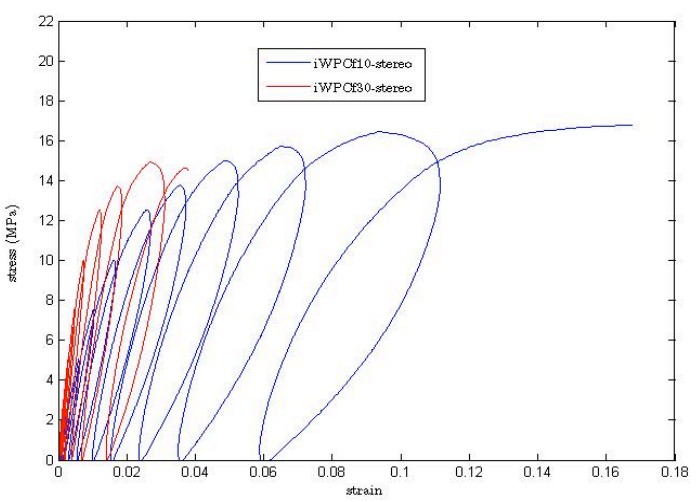

(b) iWPCf10 and iWPCf30 formulations

Fig. 4. Stress / strain cyclic tensile test curves

In the other hand, for the iWPCf10 formulation, an initial MOE value of $1155 \mathrm{MPa}$ can be evaluated. The MOE values evaluated on the three successive loading-unloading loops are respectively $1300 \mathrm{MPa}, 1349 \mathrm{MPa}$, and $1362 \mathrm{MPa}$. From the five last cycles the MOE decreases until $1245 \mathrm{MPa}$. In the same way, the MOE values calculated for the iWPCf30 formulation on the first three loops are respectively $2400 \mathrm{MPa}, 2550 \mathrm{MPa}$, and $2625 \mathrm{MPa}$, then the MOE decreases for the last two loops from $2480 \mathrm{MPa}$ to $2235 \mathrm{MPa}$. The global analysis shows that we have a non-linear behaviour of the material during the five loading-unloading cycles. One can conclude that the behaviour of the WPC can be considered as viscous during the whole experience.

\subsection{Morphology}

Microstructure of the fracture surface of iWPCf30 tensile specimens (with and without additive) is examined using SEM [4] to characterize the morphology of the wood fibre / HDPE interface. The SEM is operated at an accelerating voltage of $15 \mathrm{kV}$, spot 5.0. Figure 5(a) and 5(b) show a stretching of the HDPE matrix before the rupture on the specimen edges. During the tensile test, the longitudinal strain measured by 3-D DIC is more important on the edges. The cause of the rupture can be explained by the fact that at the beginning of the test, a stretching of the HDPE on the edges generates a weakening of the sample. Here, the wood fibre intervene to avoid the rupture. Therefore, the mechanical composite properties depend on the wood fibre content. In fact, if the fibre is oriented in the tensile direction (Figure 5(c)) a loose of fibre is observed (the fibre is pulledoff). In the other case, the fibre is broken (Figure 5(e)). In addition, the Figure 5(d) displays in the case of non-coupled composites a rough morphology presenting a hole from pull-out fibres that confirms the weakness of the interfacial bonding between the wood fibre and the HDPE matrix. Finally, morphology observation presented in Figure 5(f) shows that the wood fibre is coated with HDPE. The presence of the MAPE coupling agents changed the morphology of the materials by improving the interfacial bonding between the wood fibre and the HDPE matrix. The same results have already been observed $[4,11]$. The SEM image shows that there is no gap between wood fibre and HDPE matrix, indicating a good interface bonding. 

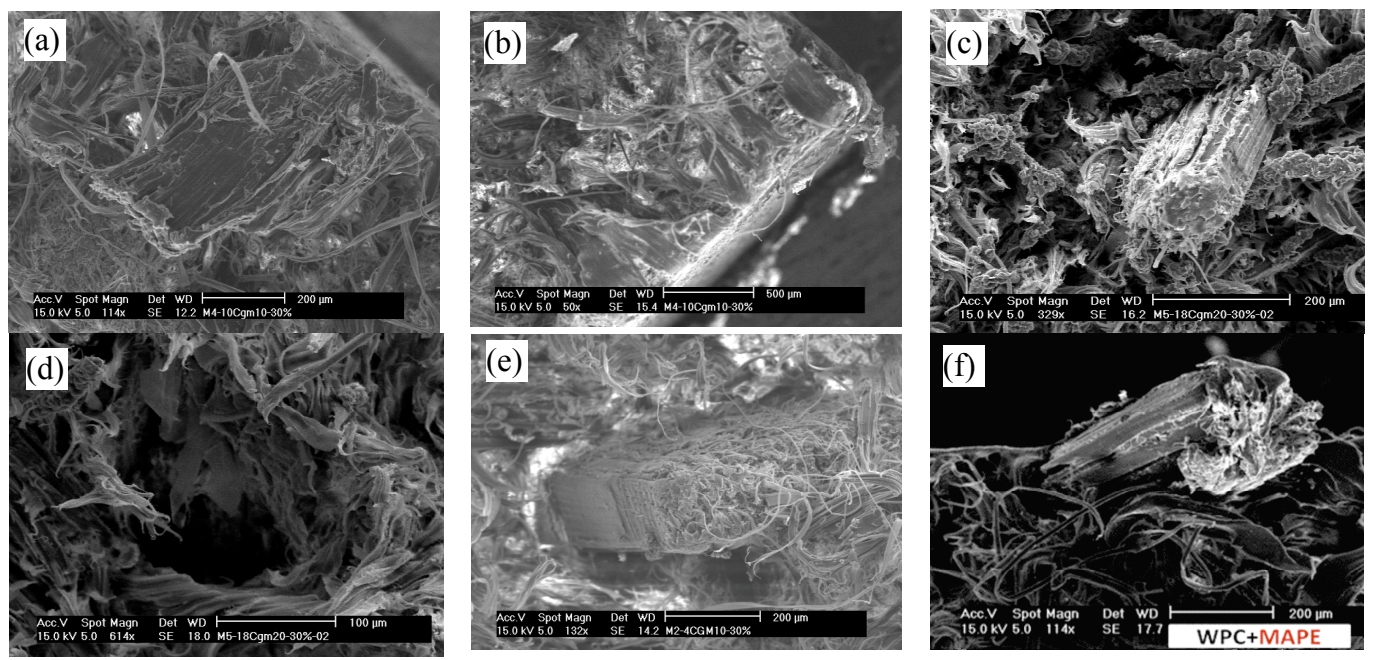

Fig. 5. SEM micrographs of fracture surfaces. (a) to (e): iWPCf30 sample, (a) 114x (b) 50x (c) 329x (d) $614 x$ (e) 132x. (f): iaWPCf30 sample at 114x

\section{Conclusion}

Tensile and 4-point bending tests were performed using the 3-D DIC technique for the strain measurements. This technique presents many advantages and allows:

- to determine a mean strain value by averaging the considered strain field, so the material heterogeneity becomes less influential;

- to observe the spatial distribution of the deformation;

- to assess the heterogeneity of the deformation during the tensile and 4-point bending experiments;

- to better understand the mechanical behaviour during the damage of WPC;

- to determine the MOE in tensile and compression during 4-point bending tests without using intrusive mechanical extensometer. (The MOE in tensile solicitation from the four-point bending tests are in good agreement with the ones obtained from direct tensile tests). In the other hand, SEM shows the important role of orientation of wood fibre in the matrix and it is confirmed that addition of MAPE improved the mechanical properties.

\section{References}

1. S. T. Georgopoulos, P.A. Tarantili, E. Avgerinos, A.G. Andreopoulos, E.G. Koukios, Polym. Deg. Stab. 90, 30 (2005)

2. C. Clemons, Forest Prod. J. 52, 10 (2002)

3. P. Nzokou, J. Freed, D.P. Kamdem, Eur. J. Wood Wood Prod. 64, 90 (2006)

4. K. Adhikary, S. Pang, M. Staiger, Comp. Part B 39, 807 (2007)

5. A. Godara, D. Raabe, I. Bergmann, R. Putz, U. Müller, Comp. Sci. Tech. 69, 139 (2009)

6. M.A. Sutton, J.-J. Orteu, H.W. Schreier, Image Correlation for Shape, Motion and Deformation Measurements, Springer (2009)

7. F. Hugot., G. Cazaurang. J. Wood Chem. Tech. 28, 283 (2008)

8. Vic-3D software, CSInc, http://www.correlatedsolutions.com/ (2009)

9. L. Robert, F. Nazaret, T. Cutard, J.-J. Orteu, Exp. Mech. 47, 761 (2007)

10. T. Ben Mbarek, L. Robert, F. Hugot, H. Sammouda, A. Graciaa, B. Charrier, J.-J. Orteu, submitted to J. Comp. Mat. (2010)

11. S.M.B. Nachtigall, G.S. Cerveira, S.M.L. Rosa, Polym. Test. 26, 619 (2007) 